\title{
‘CHEVIGNON’: NUEVA VARIEDAD DE TRIGO DE INVIERNO PARA EL SUR DE CHILE
}

\section{‘CHEVIGNON’: A NEW WINTER WHEAT CULTIVAR FOR SOUTHERN} CHILE

\author{
Claudio Jobet $^{1 \mathrm{a} *}$, Iván Matus ${ }^{2 \mathrm{a}}$, Dalma Castillo ${ }^{2 \mathrm{~b}}$, Javier Zuñiga ${ }^{1 \mathrm{~b}}$, Manuel Muñoz ${ }^{3}$, Christian Alfaro ${ }^{4}$ \\ y Juan Carlos García ${ }^{5}$
}

${ }^{1 a}$ Instituto de Investigaciones Agropecuarias (INIA), Centro Regional Carillanca, Casilla 58-D, Temuco, Chile https://orcid.org/0000-0003-1756-3523

${ }^{1 b}$ Instituto de Investigaciones Agropecuarias (INIA), Centro Regional Carillanca, Casilla 58-D, Temuco, Chile. https://orcid.org/0000-0002-7267-4472

${ }^{2 a}$ Instituto de Investigaciones Agropecuarias (INIA), Centro Regional Quilamapu, Casilla 246, Chillán, Chile https://orcid.org/0000-0003-2161-9286

${ }^{2 b}$ Instituto de Investigaciones Agropecuarias (INIA), Centro Regional Quilamapu, Casilla 246, Chillán, Chile https://orcid.org/0000-0001-7068-467X

${ }^{3}$ Instituto de Investigaciones Agropecuarias (INIA), Centro Regional Remehue, Casilla 24, Osorno, Chile https://orcid.org/0000-0002-8736-5613

${ }^{4}$ Instituto de Investigaciones Agropecuarias (INIA), Centro Regional Rayentue, Casilla 13, Rengo, Chile https://orcid.org/0000-0003-0861-3349

${ }^{5}$ Universidad de La Frontera, Facultad de Agronomía, Casilla 54-D, Temuco, Chile

* Autor para correspondencia E-mail: cjobet@inia.cl

\section{RESUMEN}

'Chevignon' es una variedad de trigo de invierno, creada por la empresa ASUR Plant Breeding de Francia e introducida a Chile por el Programa de Mejoramiento de Trigo de INIA Carillanca. Es un trigo de altura mediana, ciclo intermedio, resistente a polvillo estriado (Puccinia striiformis West. $f$. $s p$. tritici Erikss) y oidio (Blumeria graminis D.C. $f$. $s p$. tritici Marchal), moderadamente resistente a polvillo de la hoja (Puccinia triticina $f$. $s p$. tritici Erikss y Henn) y muy buena tolerancia a septoria (Mycosphaerella graminicola (Fuckel) Sand). Es una variedad de alto rendimiento, con buenas características de calidad para panificación directa. Es de hábito de desarrollo invernal, de crecimiento rastrero al estado de plántula y de buena macolla terminal. Sus hojas son de color verde pálido, con hoja bandera semi curvada. La espiga es de color blanco, semi erecta, forma paralela, densidad alta y sin barbas. El grano es de forma redondeada, tamaño mediano, color rojo, pudiendo presentar ocasionalmente ciertos cambios de pigmentación en el endosperma. La altura de planta adulta varía entre 105 y $110 \mathrm{~cm}$, con un promedio de $105 \mathrm{~cm}$, y con una moderada resistencia a la tendedura. Su período vegetativo desde siembra a espigadura es más tardío que 'Maxwell' en alrededor de cinco días y más precoz que 'Bicentenario INIA' y 'Kiron INIA' en aproximadamente 8 a 10 días, respectivamente.

Palabras clave: cultivar, trigo invierno, rendimiento, calidad. 


\section{ABSTRACT}

'Chevignon' is a winter wheat variety developed by ASUR Plant Breeding of France and introduced to Chile by the National Wheat Breeding Program at INIA Carillanca. The variety has medium plant height, intermediate cycle, with resistance to yellow rust (Puccinia striiformis West. f. $s p$. tritici Erikss), and powdery mildew (Blumeria graminis D.C. f. sp. tritici Marchal), moderate resistance to leaf rust (Puccinia triticina f. sp. tritici Erikss y Henn) and tolerance to septoria leaf blotch (Mycosphaerella graminicola (Fuckel) Sand). It is a high yielding winter wheat variety with good quality characteristics for direct baking. It has a prostrate growth habit at seedling stage, with high tillering production. Its leaves are pale green, with a semi-drooping flag leaf. The spike is white, semi-erect, and parallel in shape, compact and without awns. The grain is round, medium-sized, and pale red, with occasional variations in endosperm pigmentation. The adult plant height varies between $105 \mathrm{~cm}$ and $110 \mathrm{~cm}$, with an average of $105 \mathrm{~cm}$, and with moderate resistance to lodging. Heading growth stage occurred 5 days later than cultivar Maxwell and 8 to 10 days earlier than 'Bicentenario INIA' and 'Kiron INIA'

Key words: cultivar, winter wheat, yield, quality.

\section{INTRODUCCIÓN}

El trigo es el cultivo más importante en Chile, en términos de volumen, superficie y valor económico de la producción, con particular importancia en las regiones del sur del país, donde se concentra gran parte de su producción y superficie de siembra (INE, 2020). Este cultivo, además posee significativos componentes sociales y laborales, por su requerimiento de mano de obra que genera, como también para las numerosas empresas agrícolas en que el trigo representa un recurso esencial de rotación, producción y comercialización. De acuerdo a información publicada por ODEPA (2020), la siembra de trigo en el país alcanza las 205.036 ha, siendo las regiones de La Araucanía (82.895 ha) y la del Nuble (35.888 ha) las que concentran la mayor superficie sembrada. De igual forma, la producción triguera del país alcanza 1,3 millones de toneladas, que corresponde al $40 \%$ de la demanda interna como país, que se produce con un rendimiento promedio de 6,2 ton $\mathrm{ha}^{-1}$.

\section{MATERIALES Y MÉTODO}

\section{Material vegetal}

'Chevignon' fue introducido como variedad de la empresa ASUR Plant Breeding de Francia en el año 2016, siendo evaluado en ensayos de rendimiento preliminar en INIA Carillanca (3850'

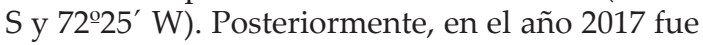
incluido en el Ensayo Estándar de Invierno en las localidades de Chillán ( $36^{\circ} 31^{\prime}$ S; 7154' O) por el norte, hasta Purranque por el sur (40 $51^{\prime}$ S; $73^{\circ} 09^{\prime}$ O), incluyendo Vilcún, y Máfil, destacándose por su tipo agronómico y adaptabilidad a zonas de siembras tempranas e inviernos largos y por su sanidad a manchas foliares (Septoria sp.).

'Chevignon' es una variedad comercial producto de una cruza realizada en Francia y liberada el año 2016 bajo el esquema de desarrollo de dobles haploides (Jobet et al., 2015). Sus progenitores son: la variedad de invierno Chevron ((Shango $x$ Macao) $x$ Tapidor) que pertenece a la empresa ASUR Plant Breeding de Francia del año 2009 y 'Boisseau', una variedad de la misma empresa de semilla liberada el año 2008. Los progenitores de 'Boisseau' corresponden a una cruza entre 'Hamac' $x$ (AARDVARK x CPB.95-71). La variedad 'Chevron' ha sido muy utilizada en programas de cruzas en INIA Carillanca, destacándose por su tipo agronómico y rendimiento. También fue una de las variedades más sembradas en Francia en los años 2010 al 2014. Por su lado, 'Hamac' es una variedad holandesa (2001), cuyos progenitores corresponden a una cruza compuesta (híbrido). Sus progenitores fueron cinco genotipos élite más dos líneas sintéticas mejoradas provenientes de la Universidad de Wageningen, en Holanda, obtenidas a partir de cruzas inter genéricas con Triticum dicoccum (AABB), especie ancestral del Triticum durum (AABB) y de Triticum aestivum (AABBDD), las cuales se utilizaron como fuente donante de genes de resistencia a enfermedades y mayor adaptabilidad a ambientes de estrés.

'Chevignon' actualmente está bajo protección europea y es la variedad más sembrada en los dos últimos años en Francia, su superficie en certificación 2019 supera las 7.000 hectáreas (ASUR Plant Breeding, comunicación personal, 2019).

\section{METODOLOGÍA}

'Chevignon' ha sido evaluado desde el año 2016 en un ensayo preliminar sin repeticiones junto a otras variedades en la localidad de Vilcún (INIA Carillanca). En respuesta a su tipo 
agronómico, características de grano y sanidad fue posteriormente incorporado, en la temporada 2017/18 al ensayo estándar nacional que incluye 20 genotipos y cinco variedades comerciales testigos, siendo estudiado desde Chillán ( $36^{\circ} 31^{\prime}$ S; $71^{\circ} 54^{\prime} \mathrm{O}$ ) hasta Purranque ( $40^{\circ} 52^{\prime}$ S; $\left.73^{\circ} 12^{\prime} \mathrm{O}\right)$, abarcando localidades geográficas con diferentes condiciones agroecológicas, como Vilcún y Máfil, las cuales comprenden cuatro regiones del sur de Chile.

La unidad experimental fue la parcela de $3 \mathrm{~m}$ de largo y 1,2 $\mathrm{m}$ de ancho (6 surcos a $0,2 \mathrm{~m}$ ), cuya superficie fue de $3,6 \mathrm{~m}^{2}$ y su diseño fue en bloques completos al azar con cuatro repeticiones. El manejo agronómico fue óptimo, con el fin de detectar diferencias entre genotipos. La fertilización base fue entre 200 a $220 \mathrm{~kg} \mathrm{ha}^{-1} \mathrm{de} \mathrm{N}$, 120 a $140 \mathrm{~kg} \mathrm{ha}^{-1}$ de $\mathrm{P}_{2} \mathrm{O}_{5}$ y entre 60 a $80 \mathrm{~kg} \mathrm{ha}^{-1}$ de $\mathrm{K}_{2} \mathrm{O}$, según la localidad y análisis de suelo. Una vez cosechadas las parcelas completas por cada localidad, se realizó un Análisis de la Varianza (ANDEVA) y para verificar las diferencias entre los promedios de los tratamientos se utilizó la Prueba de Rango Múltiple de Tukey ( $\mathrm{P} \leq$ $0,05)$. Los caracteres de calidad del grano en las temporadas 2017 y 2018 se analizaron en el Laboratorio de Calidad de Trigo en INIA Carillanca. Se determinaron: Gluten húmedo (\%), Sedimentación (\%), Proteína del grano (\%), Valor $\mathrm{W}$, entre otros.

\section{RESULTADOS Y DISCUSIÓN}

\section{Descripción morfológica de la planta}

Es un trigo de hábito de desarrollo invernal (requiere vernalización), de crecimiento muy rastrero al estado de plántula, desarrollando posteriormente una buena macolla. Sus hojas son de color verde pálido, con hoja bandera semicurvada. Presenta cierta pigmentación antociánica de débil intensidad en las aurículas. La espiga es de color blanco, semi erecta, de forma paralela, densidad alta y sin barbas. El grano es de forma redondeada, tamaño mediano, color rojo a café oscuro, pudiendo presentar ocasionalmente ciertos cambios de pigmentación en el endosperma, con cepillo ausente o muy corto, surco central marcado y un peso promedio de $48 \mathrm{~g}$ los 1000 granos, con un rango de 42 a $54 \mathrm{~g}$, dependiendo de la época de siembra y localidad. La altura de planta adulta varía entre 105 y $110 \mathrm{~cm}$, con un promedio de $105 \mathrm{~cm}$, siendo considerado un trigo de altura media, similar a la variedad de invierno Bicentenario INIA.

\section{Características agronómicas}

Tiene tallo hueco de mediana longitud, con moderada resistencia a la tendedura, similar a la variedad 'Dollinco INIA', a niveles de alta productividad es recomendable el uso de un regulador de crecimiento. Su período vegetativo desde siembra a espigadura en INIA Carillanca, es alrededor diez días más precoz que la variedad 'Bicentenario INIA'. Cuando se siembra el 20 de mayo en INIA Carillanca, espiga entre el 18 y 20 de noviembre (180 días aproximadamente de siembra a espigadura). En Purranque, sembrado el 20 de mayo, espiga alrededor del 18 de diciembre (210 días aproximadamente de siembra a espigadura), siendo alrededor de doce días más precoz que 'Bicentenario INIA'. Es un período que reúne entre 720 a 780 grados días, base $5^{\circ} \mathrm{C}$ en Vilcún, y entre 757 y 854 grados días, base $5^{\circ} \mathrm{C}$ en Purranque.

\section{Características fitopatológicas}

'Chevignon' es una variedad de trigo que ha presentado resistencia a polvillo estriado (Puccinia striiformis West. f. sp. tritici Erikss.) y moderada resistencia a polvillo colorado de la hoja (Puccinia triticina f. sp. tritici Erikss. y Henn), (Peterson et al., 1984). Tiene muy buena tolerancia a septoriosis (Mycosphaerella graminicola (Fuckel) J. Schröt.) y es resistente a oidio (Blumeria graminis D.C. f. sp. tritici Marchal), según datos observados en todas las localidades y temporadas consideradas (Saari y Prescott, 1975).

\section{Rendimiento en granos}

'Chevignon' superó, en promedio, en todas las localidades, a 'Maxi Baer', testigo oficial del Servicio Agrícola y Ganadero (SAG), y a las variedades testigos utilizados en el ensayo ('Maxwell', 'Rocky INIA' y 'Kiron INIA'). Su promedio en todas las localidades y para los tres años evaluados, fue un $10,8 \%$ superior a 'Maxwell' (15,3 t ha- ${ }^{-1}$ versus 13,8 $\mathrm{t} \mathrm{ha}^{-1}$ ); un 14,2\% superior a 'Rocky INIA' (15,3 t ha' ${ }^{-1}$ versus 13,4 t $\left.\mathrm{ha}^{-1}\right)$ y un $15,6 \%$ superior a 'Kiron INIA' $(15,3$ $\mathrm{t}$ ha $^{-1}$ versus $13,3 \mathrm{t} \mathrm{ha}^{-1}$ ) (Tabla 1 ). La diferencia más notable entre 'Chevignon' y las variedades de invierno utilizadas como testigos se presentó en la localidad de Galvarino (Región de La Araucanía), durante la temporada 2018-2019 (datos no presentados), donde las superó de forma significativa, alcanzando $20,5 \mathrm{t} \mathrm{ha}^{-1}$, cifra que supera el record mundial de rendimiento en trigo. Los niveles productivos alcanzados por 'Chevignon' observados en la mayoría de las localidades fueron superiores a los mostrados por los testigos utilizados, confirmando de esta manera el alto potencial productivo que tiene esta variedad en respuesta a las diferentes características ambientales del centro sur y sur de Chile. 
Tabla 1. Rendimiento promedio ( $\left.\mathrm{t} \mathrm{ha} \mathrm{a}^{-1}\right)$ de 'Chevignon' comparado con variedades de trigo en cuatro localidades del sur de Chile durante las temporadas 2016/17 al 2018/19.

Table 1. Mean grain yield ( $\left.\mathrm{t} \mathrm{ha} \mathrm{a}^{-1}\right)$ of 'Chevignon' compared to wheat varieties in four locations of southern Chile during the seasons 2016/17 to 2018/19.

\begin{tabular}{lccccc}
\hline Localidad & Temporada & $\begin{array}{c}\text { Rendimiento } \\
\text { Chevignon* }\end{array}$ & $\begin{array}{c}\text { Rendimiento } \\
\text { Maxwell* }\end{array}$ & $\begin{array}{c}\text { Rendimiento } \\
\text { Rocky INIA* }\end{array}$ & $\begin{array}{c}\text { Rendimiento } \\
\text { Kiron INIA* }\end{array}$ \\
\hline Chillán** & $2017 / 2018$ & $19,1 \mathrm{a}$ & $16,2 \mathrm{~b}$ & $15,7 \mathrm{~b}$ & $16,0 \mathrm{~b}$ \\
Promedio localidad & $2018 / 2019$ & $14,2 \mathrm{a}$ & $12,1 \mathrm{~b}$ & $11,9 \mathrm{~b}$ & $12,3 \mathrm{~b}$ \\
& & $\mathbf{1 6 , 9}$ & $\mathbf{1 4 , 2}$ & $\mathbf{1 3 , 8}$ & $\mathbf{1 4 , 1}$ \\
Vilcún & $2016 / 2017$ & 13,1 & 12,6 & 11,8 & 13,3 \\
& $2017 / 2018$ & $15,1 \mathrm{a}$ & $13,7 \mathrm{~b}$ & $11,7 \mathrm{c}$ & $13,6 \mathrm{~b}$ \\
Promedio localidad & $2018 / 2019$ & 11,7 & 10,8 & 9,9 & 11,4 \\
& & $\mathbf{1 3 , 2}$ & $\mathbf{1 2 , 4}$ & $\mathbf{1 1 , 1}$ & $\mathbf{1 2 , 8}$ \\
Máfil & & & & & \\
& $2017 / 2018$ & 16,9 & 16,2 & 16,4 & 16,1 \\
Promedio localidad & $2018 / 2019$ & $16,2 \mathrm{a}$ & $14,6 \mathrm{~b}$ & $13,4 \mathrm{c}$ & $14,5 \mathrm{~b}$ \\
& & $\mathbf{1 6 , 5}$ & $\mathbf{1 5 , 4}$ & $\mathbf{1 4 , 9}$ & $\mathbf{1 5 , 3}$ \\
Purranque & & & & & \\
& $2017 / 2018$ & 17,7 & 17,3 & 14,8 & 15,5 \\
Promedio localidad & $2018 / 2019$ & $16,1 \mathrm{a}$ & $13,0 \mathrm{c}$ & $12,8 \mathrm{c}$ & $14,0 \mathrm{~b}$ \\
\hline Promedio Total & & $\mathbf{1 6 , 5}$ & $\mathbf{1 5 , 1}$ & $\mathbf{1 3 , 8}$ & $\mathbf{1 4 , 7}$ \\
\hline
\end{tabular}

*Medias con las mismas letras no difieren estadísticamente entre 'Chevignon' y los testigos INIA, Tukey (P $\leq 0,05)$. ${ }^{* *}$ Con riego.

\section{Calidad industrial}

Es un trigo de buen peso hectolitro (sobre $80 \mathrm{~kg}$ $\mathrm{hL}^{-1}$ ) y grano de textura dura (Tabla 2). Presenta un porcentaje de proteína superior al $10 \%$, una cifra de sedimentación sobre $41 \mathrm{~mL}$, y su contenido de gluten húmedo alcanza a un $26 \%$, que lo ubica en la categoría de trigo intermedio (NCh 12372000) de acuerdo a INN (2000). Sus características farinográficas son aceptables, con un volumen de pan sobre $680 \mathrm{~cm}^{3}$, miga de buen color y adecuada textura (Laboratorio de Calidad de Trigo, INIA, Carillanca, 2019). Sus características promedio alveográficas (parámetros reológicos) son buenas, con un valor de trabajo $\mathrm{W}$ de 237, adecuada resistencia al estiramiento $(106,6)$, una extensibilidad de la masa (L) igual a 68,6, siendo estos valores muy superiores a los observados para la variedad Bicentenario INIA (testigo comparable), ya que la tenacidad de esta última hace limitar su condición de trigo de aptitud panadera, para lo cual se hace necesario recurrir a mezclas, lo que no debiera afectar la industrialización de esta nueva variedad de trigo. Otra característica sobresaliente de 'Chevignon' respecto a 'Bicentenario INIA' es el color de su harina, la cual es más blanca, y, por lo tanto, más aceptada por la industria molinera, característica cada vez es más demandada. 'Bicentenario INIA' ha sido exitoso en rendimiento, pero tiene ciertos reparos por parte de la industria, especialmente por su color de harina y tenacidad de masa. Ambas características han sido superadas, en parte, por esta nueva variedad, lo que constituye un gran avance, si se considera además el plus de rendir más en todas las localidades (Tabla 1).

Adicionalmente se ha medido el color de harina en distintos orígenes geográficos por medio del cromómetro marca Minolta, modelo CR400. Esta medición considera parámetros de espacio de color CIELAB e índice de color (Oliver et al., 1993). Este arrojó un valor de índice de color de 82,7 lo que significa que confiere una harina más blanca que 'Bicentenario INIA', siendo de tono más luminoso y menos amarillento.

\section{Perfil electroforético de proteínas (gluteninas) de alto peso molecular}

'Chevignon' contiene el alelo 1 en el locus Glu$1 \mathrm{~A}$, ubicado en el brazo largo del cromosoma 1A, los alelos $\mathbf{6 . 2 + 1 8}$ en el locus Glu-1B (menor tenacidad), ubicado en el brazo largo del cromosoma 1B y los alelos $\mathbf{5 + 1 0}$ en el genoma D. Esto, de acuerdo a la identificación de bandas relacionadas a gluteninas de alto peso molecular 
Tabla 2. Características de calidad de 'Chevignon' comparada con 'Bicentenario INIA'. Table 2. Quality characteristics of 'Chevignon' compared with 'Bicentenario INIA'.

\begin{tabular}{|c|c|c|c|c|c|c|}
\hline & \multicolumn{3}{|c|}{ Chevignon* } & \multicolumn{3}{|c|}{ Bicentenario INIA* } \\
\hline & 2017/18 & 2018/19 & Promedio & $2017 / 18$ & $2018 / 19$ & Promedio \\
\hline Índice de dureza $(\%)^{(1)}$ & 17,1 & 18,3 & 17,7 & 15,8 & 16,0 & 15,9 \\
\hline Peso hectolitro $\left(\mathrm{kg} \mathrm{hL}^{-1}\right)$ & 81,7 & 80,2 & 80,1 & 80,2 & 80,6 & 80,5 \\
\hline Sedimentación Zeleny $(\mathrm{mL})^{(2)}$ & 44,6 & 38,4 & 41,5 & 41,0 & 37,4 & 39,2 \\
\hline Proteína $(\mathrm{Nx} 5,7)(\%)$ & 10,4 & 10,2 & 10,3 & 8,9 & 9,1 & 9,0 \\
\hline Valor W, Alveograma ${ }^{(3)}$ & 230 & 244 & 237,0 & 226 & 175 & 200,5 \\
\hline $\mathrm{P}$ & 103,1 & 110,1 & 106,6 & 79,4 & 83,3 & 81,4 \\
\hline $\mathrm{L}$ & 69,1 & 68,0 & 68,6 & 35,0 & 32,2 & 33,6 \\
\hline $\mathrm{P} / \mathrm{L}^{(4)}$ & 1,5 & 1,6 & 1,6 & 2,3 & 2,6 & 2,5 \\
\hline Volumen de pan $\left(\mathrm{cm}^{3}\right)^{(5)}$ & 678 & 695 & 686,5 & 645 & 685 & 662,5 \\
\hline Gluten húmedo $(\%)^{(6)}$ & 26,4 & 26,8 & 26,6 & 24,4 & 25,6 & 25,0 \\
\hline Falling Number $(\mathrm{sec})^{(7)}$ & 452 & 417 & 434,5 & 325 & 357 & 341,0 \\
\hline
\end{tabular}

* Valores de las temporadas 2018 y 2019 y promedio de siembras en Carillanca (Laboratorio de Calidad de Trigo, INIA Carillanca).

(1) Índice de dureza: Valores de 16 a 25 corresponden a un grano duro; valores de 26 a 30 corresponden a un grano semiduro; valores de 31 o más corresponden a un grano blando.

(2) Sedimentación Zeleny: Cifras de 17 a $26,9 \mathrm{~cm}^{3}$ corresponden a un trigo suave; cifras de 27 a $32,9 \mathrm{~cm}^{3}$ corresponden a un trigo intermedio; cifras de 33 a $44 \mathrm{~cm}^{3}$ corresponde a un trigo fuerte; cifras superiores a $44 \mathrm{~cm}^{3}$ corresponde a un trigo muy fuerte.

(3) Valor W (Alveograma): Menor de 160 corresponde a un valor bajo; entre 161 a 250, corresponde a un valor medio; entre 251 a más, corresponde a un valor bueno.

(4) Relación P/L: Bajo 1; Trigo de adecuada tenacidad; sobre 1; Trigo tenaz; sobre 2; Trigo muy tenaz, requiere de un ingrediente que mejore su extensibilidad.

(5) Volumen de pan: Menor de 595, corresponde a un valor bajo; entre 596 y 700, corresponde a un valor bueno, valores mayores a 700, corresponden a un valor muy bueno.

(6) Igual o mayor a $30 \%$ corresponde a trigo fuerte; entre 29,9 y $25,0 \%$, corresponde a un trigo intermedio; contenido mínimo de $18,0 \%$ corresponde a un trigo suave.

(7) Falling Number: Bajo 200, trigo con Alfa amilasa activada; entre 200 a 300, trigo con poca cantidad de Alfa amilasa; sobre 300, trigo sin Alfa amilasa.

Se consideran buenos valores: ${ }^{1}$ Sobre $30 ;{ }^{2}$ Sobre los $44 \mathrm{~cm}^{3} ;{ }^{3}$ Sobre los $250 ;{ }^{4}$ Bajo $1 ;{ }^{5}$ Sobre $700 \mathrm{cc} ;{ }^{6}$ Sobre $30 \% ;{ }^{7}$ Sobre $300 \mathrm{seg}$.

establecida por Payne (1987) y Shewry et al. (1992). El score, de acuerdo con Paine (1987), para estas bandas es de ocho, lo cual la clasifica como una variedad superior en cuanto a calidad de estas proteínas, siendo el mínimo 4 y el máximo 10.

\section{ANTECEDENTES MOLECULARES}

\section{Textura de grano}

'Chevignon' contiene el alelo de dureza PIN $\boldsymbol{b}(\boldsymbol{b})$, identificado por medio de PCR (Reacción en cadena de polimerasa), lo que se confirma con el índice de dureza observado mediante espectroscopía NIR $(17,7)$ y avala el uso industrial de la variedad en la molinería (Zúñiga et al., 2001).

\section{Segmento heterólogo}

Por otra parte, no presenta elemento heterólogo de centeno, (translocación), identificado también mediante PCR (D'Oviedo y Anderson, 1995). Este ha sido confirmado también por el análisis de gliadinas, efectuado según Bushuk y Zillman (1978), el cual indica que no porta la translocación específica trigo-centeno 1BL.1RS (Zuñiga et al., 2000). La presencia de la translocación 1BL.1RS, que es la más común, se ha asociado con defectos en la calidad industrial del trigo, incluyendo la producción de masas pegajosas, propiedades de mezcla inferiores, bajo volumen de sedimentación y fermentación deficiente en trigo harinero (Li et al., 2016), así como bajo volumen de microsedimentación en trigo panadero (Oak y Tamhankar, 2016). Por otro lado, el segmento heterólogo derivado del centeno también puede producir un efecto de oscurecimiento de harina en diferentes niveles afectando de manera diferentes la expresión de la calidad industrial (Grayboch, 2001; Zhao et al., 2012). 


\section{Fotoperíodo}

Porta el gen de sensibilidad al fotoperíodo ppd-D1 (Beales et al., 2007), estableciéndose mediante análisis PCR de muestras de ADN genómico representativo de la variedad Chevignon. Esto permite que sea un trigo cuyo desarrollo es dependiente de las horas de luz recibidas, por tanto, su precocidad es similar a la de los cultivares de ciclo intermedio, como 'Maxwell'.

\section{Requerimiento de frío}

Las plantas que tienen uno o más alelos " $\mathrm{b}$ " en el gen VRN1 (Yan et al., 2004) tienen menor requerimiento de horas-frío para el inicio de la floración que las plantas de hábito invernal. De acuerdo a la metodología descrita por $\mathrm{Fu}$ et al. (2005), la variedad 'Chevignon' porta los alelos Vrn-A1(a)/Vrn-B1(a)/Vrn-D1(a); por tanto, sus requerimientos de frío son similares a un trigo de tipo invernal. Esta reacción se confirma con los resultados observados en los ensayos en época de siembra, donde se aprecia claramente que el rendimiento de 'Chevignon' va disminuyendo a medida que se atrasa la época de siembra (Datos no presentados).

\section{Tolerancia a la acidez de suelo}

'Chevignon' es un trigo sensible a la acidez de suelo y no porta genes que le confieren tolerancia a este tipo de suelo. Efectivamente, esto ha sido confirmado por los análisis moleculares (Sasaki et al., 2006)., los cuales señalan que este cultivar porta el alelo TaALMT I, relacionado con sensibilidad a la acidez, ya que ambos progenitores no tienen antecedentes de tolerancia a esta característica; por lo demás, los trigos europeos no han sido seleccionados para este parámetro, ya que sus suelos tienden a ser básicos y de $\mathrm{pH}$ relativamente neutro (Zúñiga, J. 2020. INIA Carillanca. Comunicación personal).

\section{Genes del enanismo}

'Chevignon' es un trigo que porta el alelo $\mathrm{RhtD1b}$ que corresponde a uno de los genes del enanismo relacionado al carácter altura de planta adulta (Volker Lain, 2019, Comunicación personal), anteriormente conocido como Rht2 (Knopf et al., 2008). La reducción de altura en variedades que portan este alelo es causada por una respuesta limitada de la planta a las giberelinas endógenas (Pearce et al., 2011), lo que produce entrenudos más cortos, menor estatura, mejor tolerancia a la tendedura y a la capacidad de sostener altos rendimientos en ambientes favorables (Würschum et al., 2017). Sin embargo, y a pesar de lo anterior, 'Chevignon' en ambientes de alta producción requiere del aporte de reguladores de crecimiento para evitar 'posibles tendeduras.

\section{ZONA DE CULTIVO Y ÁREA DE SIEMBRA}

'Chevignon' se recomienda desde la región del Bio Bio al sur, en localidades de inviernos largos y primaveras húmedas. Por su ciclo (más rápido que 'Bicentenario INIA'), la siembra debiera establecerse entre principios de mayo hasta el 20 de junio, aun cuando sembrado hasta fines de junio, en Vilcún, presenta un buen desarrollo y adecuada producción de espigas. Es un trigo que tiene gran potencial para la zona de los suelos trumaos y en sectores de precordillera.

\section{CONCLUSIONES}

Considerando que en las regiones del sur de Chile hay una superficie importante de suelos agrícolas dedicados a siembras de trigo, la incorporación de genética nueva con características superiores, en términos de adaptabilidad, sanidad, productividad y calidad industrial al resto de las variedades comerciales que se están utilizando, es muy importante ofrecer al agricultor una variedad superior, y satisfacer la demanda de las empresas e industrias. Del mismo modo, y en respuesta a su buena sanidad respecto a la tolerancia y resistencia a las principales enfermedades que atacan al trigo, el hecho de no tener que hacer aplicaciones excesivas de productos químicos, no solo disminuye el costo operacional, sino que impide afectar las condiciones intrínsecas de un medio ambiente más natural y armónico.

\section{LITERATURA CITADA}

Beales J., A. Turner, S. Griffiths, J.W. Snape, and D.A. Laurie. 2007. A pseudo-response regulator is misexpressed in the photoperiod insensitive Ppd-D1a mutant of wheat (Triticum aestivum L.). Theor. Appl. Genet. 115:721-733.

Bushuk, W., and R. Zillman. 1978. Wheat cultivar identification by gliadin electrophoregrams. I. Apparatus, method and nomenclature. Can. J. Plant Sci. 58:505-515.

D'Oviedo, R., and O. Anderson. 1995. PCR analysis to distinguish between alleles of a member of a multigene family correlated with bread-making quality. Theor. Appl. Genet. 88:759-763.

Fu, D.L., P. Szücs, L.L. Yan, M. Helguera, J.S. Skinner, J.V. Zitzewitz, et al. 2005. Large deletions within the first intron in $V R N-1$ are associated with spring growth habit in barley and wheat. Mol. Gen. Genomics 273:54-65. 
Graybosch R.A. 2001. Uneasy unions: Quality effects of rye chromatin transfers to wheat. Journal of Cereal Science 33(1):3-16.

INE, 2020. Cultivos anuales esenciales, superficie, producción y rendimientos, según región. Año agrícola 2019/2020. Disponible en http:// www.odepa.minagri.gob.cl (Consultado 08 julio de 2020).

INN. 2000. Norma Chilena. Nch 1237-2000. Trigo Harinero - Requisitos. 16 p. Instituto Nacional de Normalización, Santiago, Chile.

Jobet, C., I. Matus, R. Madariaga, R. Campillo, J. Zuñiga, J. Mejías, et al. 2015. Konde INIA, primera variedad doble haploide de trigo invernal para el sur de Chile. Chilean J. Agric. Anim. Sci., ex Agro-Ciencia 31(3):234-238.

Knopf, C, H. Becker, E. Ebmeyer, and V. Korzun. 2008. Occurrence of three dwarfing Rht genes in German winter wheat varieties. Cereal Research Communications 36(4):553-60.

Li, Z., T. Ren, B. Yan, F. Tan, M. Yang, and Z. Ren 2016. A mutant with expression deletion of gene Sec-1 in a 1RS.1BL line and its effect on production quality of wheat. PLoS ONE 11(1):e0146943. doi:10.1371/journal. pone. 0146943 .

Oak, M.D., and S.A. Tamhankar. 2016. 1BL/1RS translocation in durum wheat and its effect on end use quality traits. J. Plant Biochem. Biotechnol. DOI: 10.1007/s13562-016-0366-6.

ODEPA. 2020. Estadísticas Productivas. Disponible en http:/www.odepa.gob.cl/basedatos/estadisticas/produ/Agr/cultivos-psa. html. (Consultado 22 de octubre de 2020).

Oliver, J., A. Blakeney, and H. Allen. 1993. The color of flour streams related to ash and pigment contents. Journal of Cereal Science 17(2):169-182.

Payne, P. 1987. Genetics of wheat storage proteins and the effect of allelic variation on breadmaking quality. Ann. Rev. Plant. Physiol. 38:141-153.

Pearce, S., R. Saville, S.P. Vaughan, P.M. Chandler, E.P. Wilhelm, C.A. Sparks, et al. 2011. Molecular characterization of Rht-1 dwarfing genes in hexaploid wheat. Plant Physiol. 157(4):1820-31.
Peterson, R., J. Campbell, y A. Hannah. 1984. A diagrammatic scale for estimating rust intensity of leaves and stem of cereals. Can. J. Res. Sect. 26:496-500.

Saari, E., and J.M. Prescott. 1975. A scale for appraising the foliar intensity of wheat diseases. Plant Dis. Reporter 59:377-380.

Sasaki, T., P. Ryan, E. Delhaize, D. Hebb, Y. Ogihara, K. Kawaura, et al. 2006. Sequence Upstream of the Wheat (Triticum aestivum L.) ALMT1 Gene and its Relationship to Aluminum Resistance. Plant and Cell Physiology 47(10):1343 - 1354.

Shewry, P., N. Halford, and A. Tathman. 1992. Critical review article. High molecular weight subunits of wheat glutenin. J. Cereal Sci. 15:105-120.

Yan, L.L., M. Helguera, K. Kato, S. Fukuyama, J. Sherman, and J. Dubcovsky. 2004. Allelic variation at the VRN-1 promoter region in polyploid wheat. Theor.Appl. Genet.109:1677-1686.

Würschum T., S. Langer, C. Longin, M. Tucker, and W. Leiser. 2017. A modern Green Revolution gene for reduced height in wheat. 2017. The Plant Journal 92(5):892-903.

Zhao, C., F. Cui, X. Wang, S. Shan, X. Li, Y. Bao, et al. 2012. Effects of 1BL/1RS translocation in wheat on agronomic performance and quality characteristics. Field Crops Research. 127(27):79-84.

Zúñiga, J., C. Jobet, H. Campos, M. Mathías, y D. Schafer. 2001. Identificación molecular de alelos de puroindolinas en trigo (Triticum aestivum L.). p. 150. IV Encuentro Latinoamericano de biotecnología vegetal, Goiania, Brasil. 04 al 08 de junio del 2001. REDBIO. Instituto Interamericano de Cooperación para la Agricultura (IICA), Goiania, Brasil.

Zúñiga, J., C. Jobet, H. Campos, P. Rathgeb, y A. Arcos. 2000. Identificación molecular de genotipos translocados en trigo (Triticum aestivum L.). p. 56. XXXIII Reunión Anual Sociedad de Genética de Chile, Concepción, Chile. 11 al 13 de octubre del 2000. Universidad de Chile, Santiago, Chile. 